\title{
Stenting of stenotic aortopulmonary collaterals in non-surgically treated adolescent or adult patients with pulmonary atresia, ventricular septal defect and multiple aortopulmonary collaterals
}

\author{
Olejnik P, Tittel P, Venczelova Z, Masura J \\ Department of Pediatric Cardiology, National Institute of Cardiovascular Diseases, Children's Cardiac Center, \\ Bratislava, Slovakia. petoolejnik@gmail.com
}

\begin{abstract}
OBJECTIVES: To present our experience with stent implantations in non-surgically treated patients with pulmonary atresia, ventricular septal defect and stenotic aortopulmonary collaterals.

METHODS: Between April 2007 and June 2009, 6 stents in 4 patients were implanted into stenosed segments of MAPCAs. The median age and weight of patients at stent implantation were 24 years (range 13-34 years) and $58.5 \mathrm{~kg}$ (range $56-70 \mathrm{~kg}$ ), respectively.

RESULTS: All implantations were successfully performed; there were no procedure associated complications. The median diameter increase of stenosed MAPCAs was $107 \%$. The mean arterial blood saturation increased from mean $78 \%$ to $84 \%$. An early increase in the median exercise duration measured by $6 \mathrm{MWT}$ was $36 \%$. Neointimal in-stent fibroproliferation in 4/6 stents and stent fracture in 1/6 patients occured in mid-term follow-up. CONCLUSION: Percutaneous catheterizational stent implantation into stenosed segments of MAPCAs is a palliative procedure, that might improve quality of life of non-surgically treated patients with PA, VSD, MAPCAs. Neointimal in-stent fibroproliferation and stent fracture can be expected as complications in mid-term follow-up. CT-angiography is recommended to confirm these complications in progressive satO ${ }_{2}$ decrease (Fig. 3, Ref. 12). Text in PDF www.elis.sk.

KEY WORDS: stent, PA, MAPCAs, non-surgically treated patients.
\end{abstract}

\section{Introduction}

Pulmonary atresia (PA) with ventricular septal defect (VSD) and multiple aortopulmonary collaterals (MAPCAs) is a complex cyanotic congenital heart defect with severe prognosis. Corrective surgery is indicated in all neonates with PA, VSD, MAPCAs. There is still a group of patients surviving without any surgical intervention, due to hypoplastic or absent native pulmonary arteries disabling surgical correction. Pulmonary blood perfusion is ensured by MAPCAs in these patients. MAPCAs are stenotic almost in $60 \%$ of cases. Stenoses are mainly located at the origin of MAPCAs, or at the site of confluence with native pulmonary arteries (if present). The stenoses can even progress with time. They result in decrease of lung perfusion, progression of cyanosis, and NYHA grade worsening. First ever tried treatment of stenosed MAPCAs was their balloon dilatation with poor outcome due to elastic recoil of the vessel (1). First ever reported successful balloon expandable stent implantation into stenosed MAPCAs was reported by

Department of Pediatric Cardiology, National Institute of Cardiovascular Diseases, Children's Cardiac Center, Bratislava, Slovakia

Address for correspondence: P. Olejnik, MD, PhD, Department of Pediatric Cardiology, National Institute of Cardiovascular Diseases, Children's Cardiac Center, Limbova 1, SK-833 51 Bratislava, Slovakia.

Phone: +0907152641
McLeod et al in 1994 (1). They reached excercise tolerance and arterial saturation improvement in short-term follow-up. The goal of our study was to evaluate our experience with stent implantations into the stenosed segments of MAPCAs in non-surgically treated adolescent or adult patients with PA, VSD, MAPCAs at the Children's Cardiac Center in Bratislava, Slovakia.

\section{Methods}

This case reports study represents a retrospective analysis of percutaneous stent implantations $(n=6)$ into stenosed MAPCAs in non-surgically treated $(n=4)$ adolescent or adult patients (3 females and 1 male) with PA, VSD and MAPCAs at our institution between April 2007 and June 2009. Balloon-expandable, premounted, stainless steel Palmaz Genesis (PG) stents (Cordis, Johnson \& Johnson, Miami, Fl, USA), premounted on 6-10 mm diameter OptaPro ballons (Cordis Endovascular, Warren, NJ, USA) were used in all procedures. For stenting, patients with focal stenoses of aortopulmonary collaterals were chosen. These stenosed segments were recognized either by CT - angiography or by diagnostic catheterization prior to interventional procedures. Proximal and distal invasive pressures of the stenosed segment were measured before stenting. The stenting was not performed in case of systemic or near systemic pressures distally. The diameters of balloons were chosen according to the maximum diameter of the 
non-stenotic part of MAPCAs. All patients were medicated with 3 months lasting clopidogrel (1 mg/kg p.o.) therapy and permanent acetylsalicylic acid (5mg/kg p.o.) therapy after intervention. The effect of stent implantation was evaluated in short-term follow-up as followed: 1 . arterial sat $\mathrm{O}_{2}$ increase, 2 . increase of vessel diameter, 3. increase of blood pressure distally to stenoses, 4 . increase in physical excersise tolerance (6 MWT). Procedure-related complications were evaluated.

Possible complications as stent fracture (2) or in-stent neointimal proliferation (3) associated with arterial $\mathrm{satO}_{2}$ decrease were evaluated in mid-term follow-up, since April 2007 to November 2013. Chest-X ray, CT - angiography $(4,5)$ or diagnostic catheterization were used for detection of these complications. Necessity and effects of re-interventions were also analysed.

\section{Results}

Case 1

19-years old male patient with non-surgically treated PA, VSD, MAPCAs clinically presenting with central cyanosis, arterial sat $\mathrm{O}_{2}$ $72 \%$, NYHA III, and recurrent syncopes. CT-angiography delineated two segmentally stenosed MAPCAs (Fig. 1). PG2460PPS stent was implanted into right-sided MAPCA consequently (Fig. 2A). Arterial satO $\mathrm{O}_{2}$ increased from $64 \%$ to $79 \%$. Post-stenting angiography confirmed markedly improved pulmonary blood flow to right lung (Fig. 2B). The minimal stenotic diameter of MAPCA

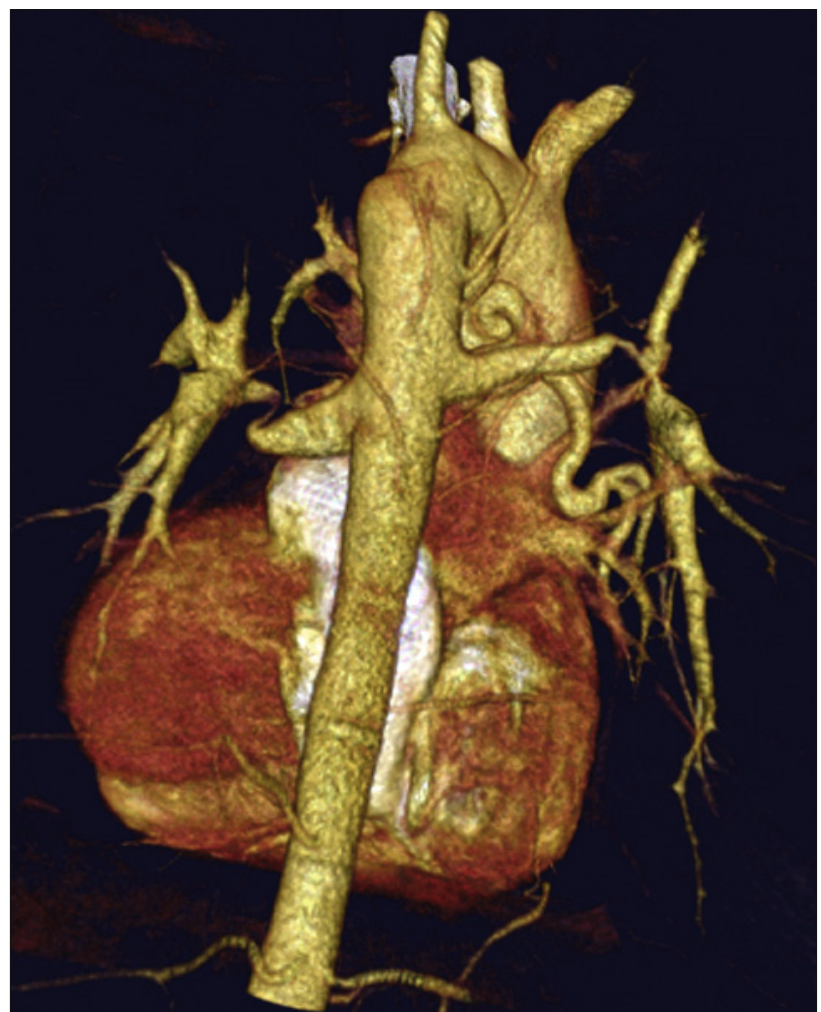

Fig. 1. Computed tomography angiography (VRT - reconstruction; posterior view) of bilateral MAPCAs with significant segmental stenoses.
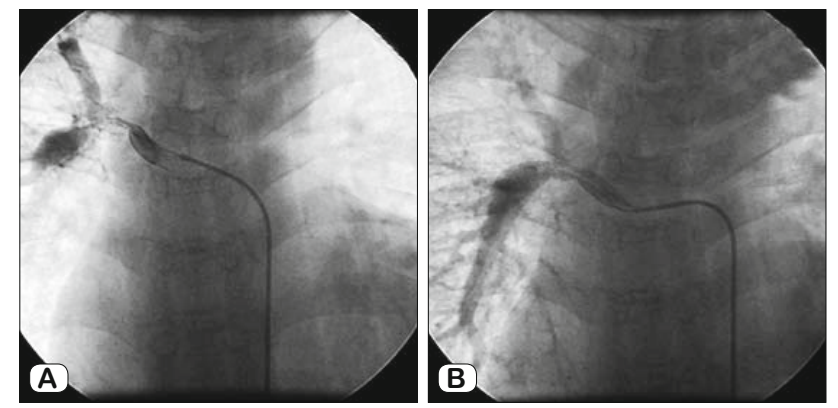

Fig. 2 Posterioanterior projection: (A) angiography of stenotic segment of right-sided MAPCA. (B) Posterioanterior projection: the final result after PG stent implantation with increased pulmonary blood perfusion in right lung segments.

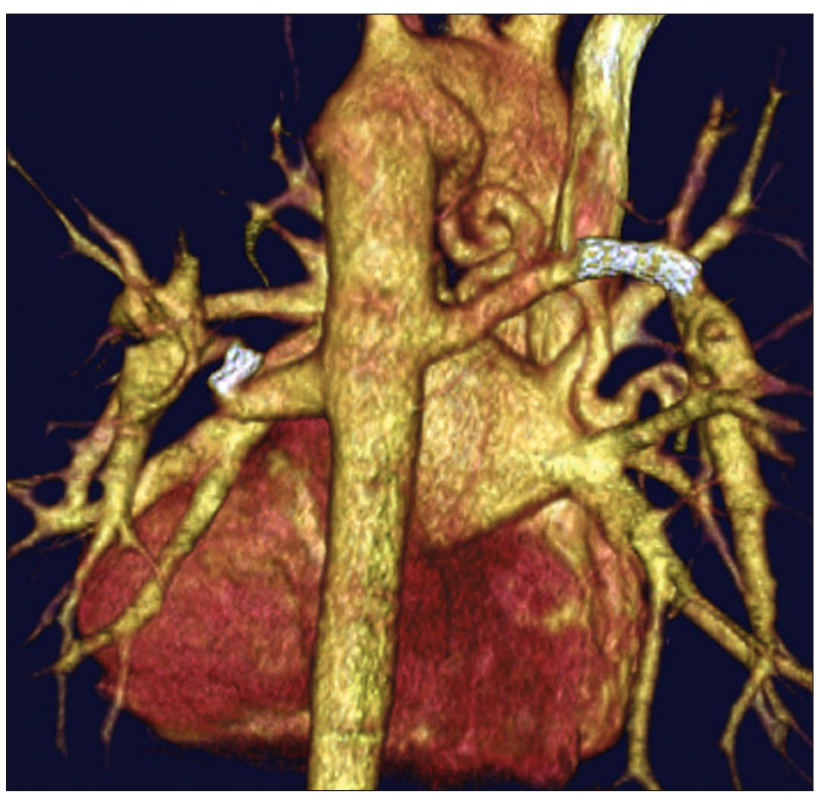

Fig. 3. Computed tomography angiography (VRT - reconstruction; posterior view) after stent implantations into stenosed segments of MAPCAs.

increased from $2.5 \mathrm{~mm}$ to $5.5 \mathrm{~mm}$. Pressures in MAPCA distally to implanted stent increased from 14/15/18 mm Hg to 28/27/26 mm Hg. 6 MWT walking distance increased from $180 \mathrm{~m}$ to 270 $\mathrm{m}$. There were no intervention related complications. Implantation of next PG 2460PPS stent into left-sided MAPCA was performed following year. Arterial $\mathrm{satO}_{2}$ increased from $76 \%$ to $82 \%$. Post-stenting angiography confirmed markedly improved pulmonary blood flow to left lung. Effective lumen of MAPCA increased from $2.2 \mathrm{~mm}$ to $5.0 \mathrm{~mm}$. Pressures in MAPCA distally to implanted stent increased from 14/13/13 mm Hg to 19/15/17 mm Hg. 6 MWT walking distance increased from $270 \mathrm{~m}$ to 400 $\mathrm{m}$. There was no intervention related complication. Effective result of both stent implantations is shown in Figure 3. Progressive arterial satO $\mathrm{O}_{2}$ decrease to minimum $66 \%$ was followed in mid-term follow-up. CTA revealed in-stent stenoses in both stents caused by neointimal fibroproliferation 5 years after first stent implantation. Angiography confirmed $4.5 \mathrm{~mm}$ effective lumen diameter in 
right-sided stent and $4 \mathrm{~mm}$ effective lumen diameter in left-sided stent, consequently. Both stents were dilated by $8 \mathrm{~mm}$ Opta Pro balloons (8-10 ATM). Stents shortened and widened to $6 \mathrm{~mm}$ diameter at the right side and $7 \mathrm{~mm}$ diameter at the left side. Subsequent angiography confirmed significant flow increase in both MAPCAs distally to implanted stents. Arterial satO $\mathrm{O}_{2}$ increased from $66 \%$ to $76 \%$. Pressures in MAPCA distally to implanted stents increased from $14 / 8 / 11 \mathrm{~mm} \mathrm{Hg}$ to $26 / 21 / 22 \mathrm{~mm} \mathrm{Hg}$ at the right side, and from 18/10/14 mm $\mathrm{Hg}$ to $26 / 20 / 23 \mathrm{~mm} \mathrm{Hg}$ at the left side. 6MWT distance increased from $200 \mathrm{~m}$ to $400 \mathrm{~m}$. There were no intervention related complications. There were no syncopes since stent implantations.

\section{Case 2}

33-years old female patient with non-surgically treated PA, VSD, MAPCAs clinically presenting with central cyanosis, arterial $\mathrm{satO}_{2} 86 \%$, and NYHA III. CT-angiography delineated two segmentally stenosed MAPCAs and two non-stenosed MAPCAs. Two PG 2460PPS stents were implanted into both stenosed MAPCAs in 1 year lapse. Arterial sat $\mathrm{O}_{2}$ increased nonsignificantly from $86 \%$ to $87 \%$ after both procedures. Effective lumen of MAPCAs increased from $3.5 \mathrm{~mm}$ to $6.0 \mathrm{~mm}$. There was no pressure change in MAPCA distally to implanted stent after intervention. 6 MWT could not be performed due to chronical neurological deficit (unilateral hemiparesis) after ischemic stroke. There was no intervention related complication. Progressive decrease of arterial satO $_{2}$ to minimum $75 \%$ was followed during mid-term follow-up. CT-angiography revealed minimal in-stent stenoses of both stents caused by neointimal fibroproliferation 5 years after first stent implantation. Effective lumen diameters were $5.5 \mathrm{~mm}$ in both stents. Both stents were dilated by $8 \mathrm{~mm}$ Opta Pro balloons (8-10 ATM). Subsequent angiography confirmed significant flow increase in both MAPCAs distally to implanted stents. Stents shortened and widened to $6.5 \mathrm{~mm}$ diameter at both sides. Arterial satO $_{2}$ increased from $75 \%$ to $84 \%$. There was no intervention related complication.

\section{Case 3}

16-years old female patient with non-surgically treated PA, VSD, MAPCAs clinically presenting with central cyanosis, arterial satO $82 \%$, and NYHA II-III. CT-angiography delineated one significantly stenosed MAPCA, and one nonstenotic MAPCA. PG 2510PPS stent was implanted into stenotic MAPCA consequently. Arterial $\mathrm{satO}_{2}$ increased from $82 \%$ to $92 \%$. Post-stenting angiography confirmed markedly improved pulmonary blood flow to right lung. Effective lumen of MAPCA increased from $4.8 \mathrm{~mm}$ to $8.0 \mathrm{~mm}$. Pressures in MAPCA distally to the implanted stent increased from 14/10/12 mm Hg to 17/14/15 mm Hg. 6MWT walking distance increased from $450 \mathrm{~m}$ to $560 \mathrm{~m}$. There was no intervention related complication. Arterial sat $\mathrm{O}_{2} 85-90 \%$ persisited during 5 years follow-up.

\section{Case 4}

13-years old female patient with non-surgically treated PA, VSD, MAPCAs clinically presenting with central cyanosis, arte- rial satO $75 \%$, and NYHA II. CT-angiography delineated two significantly stenosed MAPCAs, both with very tortuous course. PG2510PPS stent was implanted into one MAPCA consequently. Second MAPCA was intended to be stented in the future. Arterial satO $75 \%$, remained without any change. Effective lumen of MAPCA increased from $3.5 \mathrm{~mm}$ to $7.0 \mathrm{~mm}$. Pressures in MAPCA distally to implanted stent increased from mean pressure of 13 $\mathrm{mm} \mathrm{Hg}$ to $15 \mathrm{~mm} \mathrm{Hg}$. Chest X-ray and CT-angiography revealed complete fracture of implanted stent with preserved flow in distal part of MAPCA in 6 months follow-up. Effective diameter of stenotic MAPCA between fractured parts of PG stent was $3.5 \mathrm{~mm}$ again. Stabilization of fractured parts of PG stent by 30XL Andrastent (Andramed, Rutlingen, Germany) implantation mounted on OptaPro $12 \mathrm{~mm} / 4 \mathrm{~cm}$ balloon (9-10 ATM) was performed consequently. As Opta $12 \mathrm{~mm} / 4 \mathrm{~cm}$ balloon ruptured during inflation, stent dilatation with Opta $9 \mathrm{~mm} / 2 \mathrm{~cm}$ balloon (12 ATM) was completed. Ruptured balloon was easily removed without any embolization. A central waist persisted on with comparison to 8 $\mathrm{mm}$ distal stent diameters. There was no change in arterial sat $\mathrm{O}_{2}$ $75 \%$, and no signs of stent fracture on chest X-ray in 5-years follow-up (6).

\section{Results}

Between April 2007 and June 2009, PG stents $(n=6)$ were implanted into stenosed MAPCAs in patients $(n=4)$ with nonsurgically treated PA, VSD, MAPCAs. The patients ages ranged from 13 to 34 years (median 24 years), and their weight ranged from 56 to $70 \mathrm{~kg}$ (median $58.5 \mathrm{~kg}$ ).

The mean arterial sat $\mathrm{O}_{2}$ increased from $78 \%$ to $84 \%$ (range 0-15\%). The mean MAPCA`s diameter increased from $3 \mathrm{~mm}$ to 6.3 $\mathrm{mm}$ (range 2.5-4.0 mm). The mean blood pressure in distal MAPCAs increased from $13 \mathrm{~mm} \mathrm{Hg}$ to $17 \mathrm{mmHg}$ (range 2-11 mmHg). 6MWT median distance increased from $355 \mathrm{~m}$ to $455 \mathrm{~m}$ (range 20-130 m). There were no procedure associated complications.

There were stent associated complications with neccesity of reinterventions in 5/6 (83\%) implanted stents during median 5 years (range 0.5-6 years) mid-term follow-up, since April 2007 to November 2013. Fractures of 1/6 (16.7\%) implanted stents and neointimal in-stent fibroproliferation of 4/6 (66.6\%) implanted stents presenting with cyanosis worsening were recognized during follow-up.

Successful implantation of 30XL Andrastent into fractured parts of previously implanted PG stent was performed 6 months after PG stent implantation with MAPCA widening from 3.5 to 5.0 $\mathrm{mm}$ in the narrowest part. There were no signs of stent fracture on chest X-ray in 5-years follow-up. The procedure was complicated by rupture of Opta $12 \mathrm{~mm} / 4 \mathrm{~cm}$ balloon during inflation. Balloon was safely removed without any embolization.

Successful balloon dilations of 4 stenotic stents were performed in 2 patients. The median percentage of arterial satO $\mathrm{O}_{2}$ increase was $13.5 \%$ (range 12.0-15.0 \%). The median percentage of MAPCA enlargement was $24 \%$ (range 14-55\%). There was $100 \%$ walking distance increase in 6 MWT. We observed no procedure associated complications. 


\section{Discussion}

Progressive stenoses of MAPCAs in non-surgically treated patients with PA, VSD, MAPCAs are leading to arterial sat $\mathrm{O}_{2}$ decrease. Stent implantation into stenosed segments of MAPCAs is a palliative procedure improving blood perfusion through lung segments supplied by these collaterals resulting in arterial satO and excersise tolerance increase (7, 8, 9, 10, 11, 12). Redington et al reached significant arterial $\mathrm{satO}_{2}$ increase from mean $64 \%$ to $78 \%$ and an early increase in exercise duration (p < 0.01) after self-expanding stents (Wallstent, Schnieder UK Inc) $(\mathrm{n}=12)$ implantations in 11 patients (7). Brown et al presented arterial satO $\mathrm{i}_{2}$ increase from mean $75 \%$ to $82 \%$ (8) In comparision to these studies, we reached arterial satO $\mathrm{O}_{2}$ increase from mean $78 \%$ to $84 \%$, and an early increase in exercise tolerance (6 MWT mean walking distance increase from $355 \mathrm{~m}$ to 455 $\mathrm{m})$ after PG stents $(\mathrm{n}=6)$ implantations in 4 patients. Minimal satO $_{2}$ increase in 2 of our patients can be explained by sufficient blood supply to the majority of lung segments through other untouched, non-significantly stenosed MAPCAs. We also reached mean MAPCA`s diameter enlargement from $3 \mathrm{~mm}$ to $6.3 \mathrm{~mm}$, and mean blood pressure increase from $13 \mathrm{~mm} \mathrm{Hg}$ to $17 \mathrm{~mm}$ $\mathrm{Hg}$ in distal MAPCAs after stent implantation. We experienced no procedure associated complications. In comparison to our study Redington et al experienced two significant complications. Firstly, appearance of pink froth in endotracheal tube as a sign of pulmonary edema requiring possitive-pressure ventilation for 36 hours. Secondly, compression of the branch of pulmonary artery by the stent with near occlusion. Patient neccesitated surgical insertion of graft between right subclavian artery and right upper lobe artery (7).

We used stainless steel, balloon-expandable, closed-cell design PG stents in all procedures. Despite the technically demanding traverse through tortous, stenotic MAPCAs, we could complete stents deployment in all procedures. In spite of that in Redington at al. study (7), and Brown et al study (8), they could not traverse through stenotic MAPCAs by a catheter in 1/13 and 2/25 stenoses, respectively. Thus occasional impossibility of stent implantation has to be considered in severly hypoplastic/stenotic MAPCAs. We do not recommend to perform stent implantations in nonstenotic or mildly-stenotic MAPCAs with mean distal pressures $>25 \mathrm{~mm} \mathrm{Hg}$ due to high risk of pulmonary arterial hypertension accentuation in lung segments supplied by these vessels. Mean preprocedural distal MAPCAs pressure was $13 \mathrm{~mm} \mathrm{Hg}$ in our study. Pulmonary arterial hypertension, as rare complication, can occasionally develop after stent implantation into significantly stenotic MAPCAs as it is reported by Redington et al (7). Mean postprocedural distal MAPCAs pressure was $17 \mathrm{~mm} \mathrm{Hg}$ in our study. Extremly tortous course of stenotic MAPCA could have been the reason for complete rupture of 1 stent in 6 months follow-up. Chest X-ray, CT-angiography and fluorscopy could distinguish this complication (6). Successful implantation of 30XL Andrastent into fractured parts of previously implanted PG stent was performed consequently. Neointimal in-stent fibroproliferation presenting with cyanosis worsening can be expected as the mid-term follow-up complication as we experienced in 4/6 stents in spite of prophylactic acetylsalicyl acid therapy. CT-angiography was an ideal imaging tool to visualize this pathology $(4,5)$. MRI is still inferior technique due to poorer spatial resolution and higher suscseptibilty to image artefacts. Redington et al observed minimal neointimal fibroproliferation (with thin rim $<1 \mathrm{~mm}$ ) in 5/7 recatheterizations (7). Severe neointimal fibroproliferation or thrombus formation with significant cyanosis worsening was remarked only in $1 / 7$ recatheterizations (7). The reason of this lower incidence of severe neointimal in-stent fibroproliferation in this study can be shorter follow-up after stent implantation (3 to 44 months) in comparision to median 5 years folow-up of our study. Neointimal in-stent fibroproliferations of MAPCAs in our study were successfully treated by catheterizational balloon dilatation with mean $13.5 \%$ (range $12.0-15.0 \%$ ) satO $_{2}$ increase. Balloon redilatations can be expected due to recurrent neointimal in-stent fibroproliferation in the future.

\section{Conclusion}

Percutaneous catheterizational PG stent implantation into stenosed segments of MAPCAs is a palliative procedure, that might improve quality of life of non-surgically treated patients with PA, VSD, MAPCAs. We did not observe any significant procedure associated complications. Neointimal in-stent fibroproliferation and stent fracture can be expected as the complications in midterm follow-up. CT-angiography is recommended to confirm these complications in progressive $\mathrm{satO}_{2}$ decrease.

\section{Learning points}

Perform stent implantations only in severly stenosed MAPCA

Do not perform stent implantations in patients with sufficient blood supply to majority of lung segments through other untouched, non-significantly stenosed MAPCAs

Do not perform stent implantations in non-stenotic or mildlystenotic MAPCAs with mean distal pressures > $25 \mathrm{~mm} \mathrm{Hg}$

You may expect neointimal in-stent fibroproliferation, or stent fracture in mid-term post-interventional follow-up

Use CT-angiography to confirm these complications in progressive $\mathrm{satO}_{2}$ decrease

\section{References}

1. McLeod KA, Blackburn ME, Gibbs JL. Stenting of stenosed aortopulmonary collaterals: a new approach to palliation in pulmonary atresia with multifocal aortopulmonary blood supply. Br Heart J 1994; 71 (5): 487-489.

2. Breinholt JP, Nugent AW, Law MA, Justino H, Mullins CE, Ing FF. Stent fractures in congenital heart disease. Catheter Cardiovasc Interv 2008; 72: 977-982. doi: 10.1002/ccd.21742.

3. Xu N, Zhang J, Li M, Pan J, Lu Z. Incidence and classification of neointimal proliferation and in-stent restenosis in post-stenting patients at 1-year interval: findings from non-invasive coronary computed tomography angiography.Eur J Radiol 2014; 83 (10): 1816-1821. doi: 10.1016/j. ejrad.2014.07.015. 
72-76

4. Eichhorn JG, Long FR, Hill SL, Cheatham JP. Multislice computed tomography as an adjunct to the management of an instent stenosis in an infant with congenital heart disease: Imaging for the future. Catheter Cardiovasc Interv 2006; 67: 477-481.

5. Olejnik P, Boruta P, Glezlova A, Bordáčová L, Mašura J. The first experiences with the high-resolution multi-slice CT-angiography in pediatric cardiology in Slovakia. Bratisl Lek Listy 2008; 109 (5): 220-223.

6. Venczelova Z, Tittel P, Masura J. First experience with AndraStent XL implantation in children and adolescents with congenital heart diseases. Catheter Cardiovasc Interv 2013; 81 (1): 103-110. doi: 10.1002/ccd.24505.

7. Redington AN, Somerville J Stenting of Aortopulmonary Collaterals in Complex Pulmonary Atresia. Circulation 1996; 94 (10): 2479-2484.

8. Brown SC, Eyskens B, Mertens L, Dumoulin M, Gewillig M. Percutaneous treatment of stenosed major aortopulmonary collaterals with balloon dilatation and stenting: what can be achieved? Heart 1998; 79: 24-28.
9. El-Said HG, Clapp S, Fagan TE, Conwell J, Nihill MR. Stenting of stenosed aortopulmonary collaterals and shunts for palliation of pulmonary atresia/ventricular septal defect. Catheter Cardiovasc Interv 2000; 49: 430-436.

10. Vance MS. Use of Palmaz stents to palliate pulmonary atresia with ventricular septal defectand stenotic aortopulmonary collaterals, Cathet Cardiovasc Diagn 1997; 40 (4): 387-389.

11. Vimala J, Kulkarni S. Stenting stenosed aortopulmonary collateral arteries in pulmonary atresia with ventricular septal defect. Indian Heart J 2004; 56 (3): 242-244.

12. Heran M, LeBlanc J. Decision-making in unoperated adults with congenital heart disease: a difficult task. Interact CardioVasc Thorac Surg 2007; 6: 820-822.

Received April 30, 2015. Accepted August 18, 2015. 